\title{
Compostaje del residuo papelero aplicado al cultivo de maíz
}

\author{
Wastepaper composting applied to corn cultivation \\ Compostagen de resíduos de papel aplicada ao cultivo de milho
}

\author{
Juan Velásquez-Barbachán \\ jvelasquezb@unap.edu.pe \\ https://orcid.org/0000-0002-4914-8407 \\ Universidad Nacional del Altiplano de Puno, Puno-Perú
}

Artículo recibido 3 de septiembre 2021 / Arbitrado y aceptado 8 de octubre 2021 / Publicado 13 de diciembre 2021

\section{RESUMEN}

La industria del papel proveniente de celulosa tiene un crecimiento de $2.5 \%$ anual, sin embargo, existe la tendencia creciente del uso de papel reciclado para los procesos de fabricación. Durante este proceso se genera un residuo papelero que no tiene una disposición final adecuada hecho que contribuye a la contaminación ambiental. El objetivo del trabajo es establecer una formulación de compost de residuo papelero que pueda aplicarse al cultivo de maíz, evaluando su rendimiento como alternativa para la gestión de residuos de la industria papelera. El diseño experimental utilizado fue de bloques completamente al azar con cuatro tratamientos $\mathrm{T} 1: 15$ t ha-1; T2:20 t ha-1; T3:25 t ha-1; T4:30 t ha-1 y cuatro repeticiones. No existió diferencia significativa en la relación raíz/vástago a los 40, 80 y 120 días después de la siembra, así como en el número de mazorcas por planta. Por otro lado, en el peso de mazorcas por planta, el rendimiento de forraje verde y rendimiento de materia seca el T4 presentó diferencia significativa. En conclusión, el compostaje en una relación 3:1 de residuo papelero y estiércol de vaca puede ser una opción viable en la gestión de residuos provenientes de la industria papelera ya que tiene efectos sobre el rendimiento de maíz.

Palabras clave: Compost; Estiércol de vaca; Gestión de residuos; Maíz; Residuo papelero
ABSTRACT

The pulp paper industry has a growth rate of $2.5 \%$ per year; however, there is a growing trend towards the use of recycled paper in the manufacturing process. During this process, a paper waste is generated that does not have an adequate final disposal, a fact that contributes to environmental pollution. The objective of this work is to establish a compost formulation of paper residue that can be applied to the corn crop, evaluating its performance as an alternative for the management of paper industry residues. The experimental design used was a completely randomized block design with four treatments $\mathrm{T} 1: 15$ t ha- $1 ; \mathrm{T} 2: 20$ t ha-1; T3:25 t ha-1; T4:30 t ha-1 and four replicates. There was no significant difference in the root/shoot ratio at 40 , 80 and 120 days after sowing, as well as in the number of ears per plant. On the other hand, in the weight of ears per plant, green forage yield and dry matter yield, T4 presented a significant difference. In conclusion, composting in a 3:1 ratio of paper residue and cow manure can be a viable option in the management of residues from the paper industry since it influences corn yield.

Key words: Compost; Corn; Cow manure; Paper waste; Waste management

\section{RESUMO}

A indústria de papel de celulose está crescendo a $2,5 \%$ ao ano, no entanto, há uma tendência crescente para o uso de papel reciclado no processo de fabricação. Durante este processo é gerado um resíduo de papel que não é descartado adequadamente, o que contribui para a poluição ambiental. $O$ objetivo deste trabalho é estabelecer uma formulação compostada de resíduos de papel que possa ser aplicada ao cultivo de milho, avaliando seu desempenho como uma alternativa para o gerenciamento de resíduos na indústria de papel. $O$ projeto experimental utilizado foi um bloco completamente aleatório com quatro tratamentos T1:15 t ha-1; T2:20 t ha-1;T3:25 tha-1; T4:30 tha-1 e quatro réplicas. Não houve diferença significativa na relação raiz/roda aos 40 , 80 e 120 dias após a semeadura, bem como no número de espigas por planta. Por outro lado, no peso das espigas por planta, no rendimento de forragem verde e no rendimento de matéria seca, - T4 mostrou diferenças significativas. Em conclusão, a compostagem em uma proporção de 3:1 de resíduos de papel para esterco de vaca pode ser uma opção viável no gerenciamento de resíduos da indústria de papel, pois tem um efeito no rendimento do milho.

Palavras-chave: Compostagem; Resíduos de papel; Esterco de vaca; Gestão de resíduos; milho 


\section{INTRODUCCIÓN}

La industria de papel genera gran cantidad de residuos sólidos que proceden de sus operaciones industriales (1). Entre los residuos sólidos más importantes sobresalen lodos de destintado procedentes del papel reciclado, residuos procedentes de la clasificación del papel, desechos de fibras obtenidos por separación mecánica y lodos del tratamiento in situ de efluentes (2); los cuales generan una gran preocupación para su adecuado tratamiento.

Las tecnologías actuales de tratamiento en países desarrollados se basan principalmente en la producción de energía a través de procesos de incineración, gasificación, pirolisis, digestión anaeróbica y biodiésel (3), no obstante, en países latinoamericanos estos residuos papeleros no tienen un tratamiento claro (4), en algunos casos son colocados en vertederos municipales, mientras que en otros son dispuestos en lugares informales atentando contra el medio ambiente.

Sin embargo, las empresas latinoamericanas de la industria de la del papel ante la presión social por el cuidado y conservación ambiental se están enfocando en prácticas ambientales sostenibles como la reutilización de residuos en sus procesos industriales (5), hecho que ha significado el uso de hasta el $40 \%$ del papel reciclado en su proceso productivo (6), permitiendo un ahorro energético y económico (7), enfocado en la gestión adecuada de residuos.

En la actualidad la gestión de residuos se ha convertido en un reto medio ambiental global (8), no solo en países desarrollados sino en países subdesarrollados, que promueve la conversión de los desechos en productos con valor agregado (9), los cuales aparte de ser económicos, son menos tóxicos (10). Sin embargo, las tecnologías actuales son costosas de implementar por las empresas, debido a ello una opción económica y viable es el compostaje.

El compostaje es una opción que permite la estabilización y biotransformación de diferentes materias primas en productos degradados(11), queademáspuedenaplicarsea la agricultura (12). Así, el compost convencional utilizado en campos de cultivo es producto de la mezcla de residuos orgánicos con estiércol de animales, mientras que el vermicompostaje es el uso de lombrices californianas (Eisenia fétida) que descomponen la materia orgánica en condiciones específicas.

Existen estudios en los cuales se ha utilizado el residuo papelero en procesos de compostaje. En una relación 1:1 de residuo papelero con estiércol de cerdo por 56 días es suficiente para lograr un compost maduro (13). De otro lado, la relación de 5:4:1 de residuo papelero más estiércol de vaca y paja resultó en una formulación eficiente para residuos orgánicos de papelera (14); de igual forma la relación 2:1:1 de lodos de papelera con estiércol de vaca y abono verde mejoró las características físicas del suelo por el contenido de Fosforo y Potasio disponible para las plantas (15); además las mezclas de lodos papeleros con residuos de té y estiércol favorecieron la sostenibilidad en el empleo de estos residuos (16). 
Por ello, el objetivo de este estudio es establecer una formulación de compost de residuo papelero con estiércol de vaca que puedan aplicarse al cultivo de maíz, evaluando su rendimiento como alternativa para la gestión de residuos de la industria papelera.

\section{MATERIALES Y MÉTODOS}

La investigación se realizó en la localidad de La Joya (con localización geográfica de $16^{\circ}$ 30' 50" S y 7150' 33" O, a 1531 m.s.n.m.), del distrito de La Joya, provincia y departamento de Arequipa, en la campaña agrícola 20192020. Durante el experimento la temperatura media anual fue de $20.1^{\circ} \mathrm{C}$, humedad relativa de $40-55 \%$ y suelos de textura arena franca. Se utilizó 3 kg de semilla de maíz hibrido PM212, producido por el Programa Cooperativo de Investigaciones en Maíz de la Universidad Agraria La Molina de Lima, Perú (17).

Para las formulaciones de compost se utilizó un residuo papelero de la producción de papeles absorbentes reciclados provenientes de la empresa Papelera Panamericana S.A., del departamento de Arequipa - Perú, al que previamente se le realizó una caracterización fisicoquímica para determinar materia orgánica, fósforo, potasio, magnesio y calcio. Se recolectaron dos toneladas de residuo papelero a los que se les aplico un preprocesamiento de secado hasta llegar al $55 \%$ de humedad, luego se realizaron dos pilas de compostaje que consistían en capas de residuo papelero con estiércol de vaca en una relación 3:1, dispuestos en un área de 6 $\mathrm{m}^{2}$. En el centro de la pila se ubicó dos tubos centrales perforados, distanciados uno del otro en un metro, los cuales funcionaban como respiraderos. El compostaje se realizó por un periodo de tres meses, en los cuales la frecuencia de riego fue interdiaria, realizando volteos semanales de la pila de compostaje. Al tercer mes se cubrió la pila de compostaje con plástico transparente hasta llegar a una humedad del 30\%. Finalmente, el compost Luego se trasladó a campo donde se le aplico a los diferentes tratamientos a fondo de surco.

Se utilizó el diseño de Bloques Completamente al Azar (DBCA), conformado por 4 tratamientos y con 4 repeticiones. Los tratamientos estuvieron compuestos por 4 niveles de compost del residuo papelero aplicado al cultivo de maíz T1: $15 \mathrm{t} \mathrm{ha}^{-1}, \mathrm{~T} 2: 20 \mathrm{t}$ $\mathrm{ha}^{-1}, \mathrm{~T} 3: 25 \mathrm{t} \mathrm{ha}^{-1}, \mathrm{~T} 4: 30 \mathrm{t} \mathrm{ha}^{-1}$, constituyendo en total 20 parcelas de 8 surcos con una longitud de $6,4 \mathrm{~m}$ de largo y $0.60 \mathrm{~m}$ entre ellos, con área por parcela de $31 \mathrm{~m}^{2}$ y área total de $824 \mathrm{~m}^{2}$.

La siembra del maíz PM-212 se realizó el 5 de octubre del 2019 aplicando el sistema de siembra manual por golpe (con 3 semillas/ golpe), a un lado del surco. La fertilización empleada fue de 160-80-0 unidades de NPK, respectivamente. En todas las parcelas se realizó el control eficiente de plagas como el "cogollero" (Spodoptera frugiperla Smith) y gusano de tierra (Feltia experta Walker). Se realizó un aporque manual a los 40 días después de la siembra. Las variables dependientes del estudio fueron relación raíz/vástago a los 40, 80 y 120 días después de la siembra, cantidad de mazorcas por planta, peso de mazorcas por planta, rendimiento de forraje verde, 
rendimiento de materia seca, los cuales se complementaron con un análisis fisicoquímico del suelo. La cosecha se realizó el 17 de febrero del 2020, cuando el cultivo cumplió 135 días de ciclo vegetativo, es decir 4.5 meses. Se utilizó el paquete estadístico SAS con un análisis de varianza en los datos cuantitativos y se procedió a realizar la prueba de medias de Tukey con un nivel de significancia del $5 \%$.

\section{RESULTADOS Y DISCUSIÓN}

\section{Caracterización fisicoquímica del residuo de papel reciclado}

Los resultados de la materia orgánica (MO), potasio $(\mathrm{K})$, magnesio $(\mathrm{Mg})$ y calcio $(\mathrm{Ca})$ concuerdan con las características de otros residuos papeleros en cuanto a la relación de abundancia y mas no en cantidad (Tabla 1). Así el Ca es el más abundante, seguido del Mg y el $\mathrm{K}(18)$, esto se explicaría por la similitud de los procesos industriales en las fábricas de papel. En el caso del fosforo (P) no se han encontrado reportes similares, sin embargo, otro autor refiere que se adicionaría este elemento durante la fabricación para mejorar la biomasa del papel (19).

Tabla 1. Análisis químico del residuo papelero.

\begin{tabular}{cc}
\hline Parámetro & Resultado \\
\hline Materia orgánica (MO) & $28.60 \%$ \\
Fosforo (P) & $234 \mathrm{mg} \mathrm{kg}^{-1}$ \\
Potasio (K) & $458 \mathrm{mg} \mathrm{kg}^{-1}$ \\
Magnesio (Mg) & $1110 \mathrm{mg} \mathrm{kg}^{-1}$ \\
Calcio (Ca) & $2080 \mathrm{mg} \mathrm{kg}^{-1}$ \\
\hline
\end{tabular}

Relación raíz/vástago a los 40, 80 y 120 días después de la siembra

No existió diferencia significativa entre los tratamientos en la variable relación raíz / vástago a los 40, 80 y 120 días después de la siembra (Tabla 2). A los 40 y 120 días el T4 presenta mayores valores en la dinámica de crecimiento de las plantas, ello puede explicarse porque a mayor nivel de compost hay mayor enraizamiento, crecimiento radicular y más biomasa en la raíz, sobre todo en el crecimiento de raíces secundarias (20); además la relación raíz/vástago muestra una tendencia inversa a la relación de $\mathrm{Ca}, \mathrm{Mg}$ y $\mathrm{K}$ del suelo (21). 
Tabla 2. Relación raíz /vástago a los 40, 80 y 120 días, cantidad y peso de mazorcas, rendimiento de forraje verde y seco.

\begin{tabular}{cccccccc}
\hline & \multicolumn{2}{c}{ RELACIÓN VÁSTAGO/RAíZ (DÍAS) } & \multicolumn{2}{c}{ MAZORCAS (GR) } & \multicolumn{2}{c}{ FORRAJE (KG) } \\
Tratamiento & $\mathbf{4 0}$ & $\mathbf{8 0}$ & $\mathbf{1 2 0}$ & Cantidad & Peso & Verde & Seco \\
\hline T0 & $0.27^{\mathrm{a}}$ & $0.49^{\mathrm{a}}$ & $0.32^{\mathrm{a}}$ & $1.29^{\mathrm{a}}$ & $414.68^{\mathrm{b}}$ & $20.80^{\mathrm{b}}$ & $10.88^{\mathrm{b}}$ \\
T1 & $0.29^{\mathrm{a}}$ & $0.64^{\mathrm{a}}$ & $0.38^{\mathrm{a}}$ & $1.29^{\mathrm{a}}$ & $348.10^{\mathrm{b}}$ & $17.13^{\mathrm{b}}$ & $9.33^{\mathrm{b}}$ \\
T2 & $0.32^{\mathrm{a}}$ & $0.56^{\mathrm{a}}$ & $0.48^{\mathrm{a}}$ & $1.21^{\mathrm{a}}$ & $341.95^{\mathrm{ab}}$ & $15.15^{\mathrm{ab}}$ & $8.45^{\mathrm{ab}}$ \\
T3 & $0.31^{\mathrm{a}}$ & $0.53^{\mathrm{a}}$ & $0.43^{\mathrm{a}}$ & $1.11^{\mathrm{a}}$ & $299.78^{\mathrm{ab}}$ & $13.60^{\mathrm{ab}}$ & $7.48^{\mathrm{ab}}$ \\
T4 & $0.37^{\mathrm{a}}$ & $0.52^{\mathrm{a}}$ & $0.49^{\mathrm{a}}$ & $1.11^{\mathrm{a}}$ & $297.30^{\mathrm{a}}$ & $12.93^{\mathrm{a}}$ & $7.38^{\mathrm{a}}$ \\
\hline
\end{tabular}

a, b Superíndices diferentes dentro de columnas indican diferencia significativa $(p<0,05)$.

T0: 0 t ha-1; T1: 15 t ha-1; T2: 20 t ha-1; T3: 25 t ha-1; T4: 30 t ha-1

\section{Número y peso de mazorcas por planta}

En la cantidad de mazorcas por planta no existe diferencia significativa entre los tratamientos (Tabla 2). Esto se explicaría porque el maíz Hibrido PM-212 puede producir más de una mazorca por planta, pero depende de las condiciones climáticas (18), además el número de mazorcas en maíces híbridos es difícil de alterar (22). En referencia al peso de las mazorcas, el T4 obtuvo diferencia significativa con los demás tratamientos. Esto podría explicarse por la mayor cantidad de compost, que mejora las características físicas del suelo (23), pero que es $50 \%$ menos efectivo que una fertilización química debido a la rápida absorción por las raíces (24).

\section{Rendimiento de forraje verde y materia seca}

Existió diferencia significativa entre el T4 y los demás tratamientos (Tabla 2). Esto se explicaría por la calidad del compost, el cual tiene la capacidad de almacenamiento de nutrientes y la liberación lenta de los mismos que incide en la capacidad de intercambio catiónico del suelo (25). Así mismo el compost de residuo papelero se utiliza como enmienda para los suelos (26), influenciando sobre el rendimiento del cultivo de maíz (25).

\section{Análisis químico del suelo}

El contenido de materia orgánica, fosforo (P) y la conductividad eléctrica se incrementó en todos los tratamientos, mientras que el potasio (K) y la capacidad de intercambio catiónico disminuyo y $\mathrm{pH}$ se mantuvo estable (Tabla 3). El ligero incremento de la materia orgánica en el suelo se explicaría por la adhesión del residuo papelero al estiércol de vaca, que facilita la biodisponibilidad de nutrientes para los microorganismos del suelo (24). Además, los cambios térmicos que ocurren durante el proceso de compostaje favorecerían el incremento de fosforo disponible (P) (27). La conductividad eléctrica estaría afectada por sales disueltas del suelo que interactúan de manera directa con el compost pero que indican una salinidad leve (28). De otro la CIC estaría afectada por la mínima cantidad de arcilla que presentan los suelos francos arenosos, reportados en forma similar en otros estudios (29), pero que también estaría siendo afectado por el $\mathrm{pH}$ del suelo (30). 
Tabla 3. Análisis químico del suelo según tratamientos.

\begin{tabular}{ccccccc}
\hline Tratamiento & $\begin{array}{c}\text { Materia } \\
\text { orgánica (\%) }\end{array}$ & $\begin{array}{c}\mathbf{P} \\
(\mathbf{p p m})\end{array}$ & $\begin{array}{c}\mathbf{K} \\
(\mathbf{p p m})\end{array}$ & $\mathbf{C . E .}(\mathbf{d s} / \mathbf{m})$ & $\mathbf{p H}$ & $\begin{array}{c}\text { CIC } \\
\text { meq/100grs }\end{array}$ \\
\hline T0 & 0.89 & 4.55 & 387.47 & 0.25 & 7.62 & 8.50 \\
T1 & 0.98 & 21.84 & 299.98 & 0.70 & 7.53 & 6.26 \\
T2 & 0.98 & 23.59 & 299.98 & 0.82 & 7.44 & 6.26 \\
T3 & 1.24 & 19.22 & 319.98 & 0.68 & 7.54 & 5.92 \\
T4 & 0.80 & 21.84 & 319.98 & 0.72 & 7.56 & 6.56 \\
\hline
\end{tabular}

P: Fosforo, K: Potasio, C. E: conductividad eléctrica, CIC: capacidad de intercambio catiónico.

\section{CONCLUSIONES}

La relación 3:1 del residuo papelero con estiércol de vaca influye sobre el peso de las mazorcas, mayor rendimiento de forraje verde y materia seca; así como en las características químicas del suelo respecto al contenido de materia orgánica, contenido de fosforo, potasio, conductividad eléctrica, $\mathrm{pH}$ y capacidad de intercambio catiónico. Se recomienda continuar las investigaciones con varios tipos de estiércoles con desperdicios de papel y el efecto sobre otros cultivos agroindustriales.

\section{Agradecimientos}

J.P. Velásquez reconoce el apoyo financiero del Proyecto Concytec-Banco Mundial "Mejoramiento y Ampliación de los Servicios del Sistema Nacional de Ciencia Tecnología e Innovación Tecnológica" 8682-PE, a través de su unidad ejecutora ProCiencia contrato número 01-2018-FONDECYT/BM-Programas de Doctorados en Áreas Estratégicas y Generales.
REFERENCIAS BIBLIOGRÁFICAS

1. Monte MC, Fuente E, Blanco A, Negro C. Waste management from pulp and paper production in the European Union. Waste Manag. 2009; 29(1):293-308. http://dx.doi.org/10.1016/j. wasman.2008.02.002

2. Rivera J. Viabilidad del proceso de gasificacion de residuos con alto contenido en material plástico. España: Universidad de Valladolid; 2018. 1-260 p. http://uvadoc.uva. es/handle/10324/33067

3. Gopal PM, Sivaram NM, Barik D. Paper industry wastes and energy generation from wastes. Energy from Toxic Organic Waste for Heat and Power Generation. India. Elsevier Ltd.; 2018; 83-97 p. https://doi.org/10.1016/ B978-0-08-102528-4.00007-9

4. Kaza S, Bhada-Tata P. Decision Maker's Guides for Solid Waste Management Technologies. Decis Maker's Guid Solid Waste Manag Technol. Estados Unidos. Worldbank. 2018. https://openknowledge.worldbank.org/ handle/10986/31694

5. Moraes DM, Silva PH, Simon I, Anholon R, Quelhas OLG, Farias JR, et al. Environmentallyresponsible corporate: Actions analysis of Latin American pulp and paper industry. Environ Challenges. 2021;4(May):100153. https://doi. org/10.1016/j.envc.2021.100153

6. Alshehrei F, Ameen F. Vermicomposting: A management tool to mitigate solid waste. 
Saudi J Biol Sci. 2021;28(6):3284-93. https://doi. org/10.1016/j.sjbs.2021.02.072

7. Devichi Wibowo I, Purwanto P, Suherman S. Solid waste management in the paper industry. E3SWeb Conf. 2020;202(November):1-6. https:// doi.org/10.1051/e3sconf/202020206026

8. Guo X, Huang J, Lu Y, Shan G, Li Q. The influence of flue gas desulphurization gypsum additive on characteristics and evolution of humic substance during co-composting of dairy manure and sugarcane pressmud. Bioresour Technol. 2016; 219:169-74. http:// dx.doi.org/10.1016/j.biortech.2016.07.125

9. Esmaeilian B, Wang B, Lewis K, Duarte F, Ratti C, Behdad S. The future of waste management in smart and sustainable cities: A review and concept paper. Waste Manag. 2018; 81:177-95. https://doi.org/10.1016/j.wasman.2018.09.047

10. Vaverková MD, Elbl J, Voběrková $S$, Koda E, Adamcová D, Mariusz Gusiatin Z, et al. Composting versus mechanical-biological treatment: Does it really make a difference in the final product parameters and maturity. Waste Manag. 2020; 106:173-83. https://doi. org/10.1016/j.wasman.2020.03.030

11. Onwosi CO, Igbokwe VC, Odimba JN, Eke IE, Nwankwoala MO, Iroh IN, et al. Composting technology in waste stabilization: On the methods, challenges and future prospects. J Environ Manage. 2017; 190:140-57. http:// dx.doi.org/10.1016/j.jenvman.2016.12.051

12. Lee LH, Wu TY, Pui K, Shak Y, Lim SL, Ng KY, et al. Sustainable approach to biotransform industrial sludge into organic fertilizer via vermicomposting: A mini-review. J Chem Technol Biotechnol. 2018;(93):925-235. https:// doi.org/10.1002/jctb.5490

13. Wong JWC, Karthikeyan OP, Selvam A. Biological nutrient transformation during composting of pig manure and paper waste. Environ Technol (United Kingdom). 2017;38(6):754-61. http://dx.doi.org/10.1080/0 9593330.2016.1211747

14. Ganguly RK, Chakraborty SK. Valorisation of toxic paper mill waste through vermicomposting: An insight towards cleaner engineering through alleviation of wastes. Clean Eng Technol. 2021;2(October 2020):100070. clet.2021.100070

15. Karmegam N, Vijayan $P$, Prakash $M$, John Paul JA. Vermicomposting of paper industry sludge with cowdung and green manure plants using Eisenia fetida: A viable option for cleaner and enriched vermicompost production. J Clean Prod. 2019; 228:718-28. https://doi. org/10.1016/j.jclepro.2019.04.313

16. Kumar V, Singh S, Singh B. Biotransformation of paper mill sludge and tea waste with cow dung using vermicomposting. Bioresour Technol. 2020; 318(August):124097. https://doi. org/10.1016/j.biortech.2020.124097

17. Lizarja Y. Comportamiento de híbridos triples de maiz amarillo duro (Zea mays L) en condiciones edafoclimaticas de costa central. Lima, Peru. Universidad Nacional Hermilio Valdizan Huánco; 2017. http://repositorio. unheval.edu.pe/handle/UNHEVAL/2864

18. Curnoe WE, Irving DC, Dow CB, Velema G, Unc A. Effect of Spring Application of a Paper Mill Soil Conditioner on Corn Yield. Agron J. 2006; 98:423-9. https://www.researchgate.net/ publication/250104405_Soil_Properties_and_ Crop_Yields_in_Response_to_Mixed_Paper_ Mill_Sludges_Dairy_Cattle_Manure_and_ Inorganic_Fertilizer_Application

19. Lillo F. Estimar el balance de fósforo en el proceso de fabricación de celulosa de fibra corta: evaluación de la carga de fósforo aportado al río Biobío. Concepcion, Chile. 2013. http://www.eula.cl/giba/wp-content/ uploads/2017/09/tesis-felipe-lillo-2013.pdf

20. Reyes J, Rueda R, Martinez D, De Limas G. Variación fenotípica de maíz. Rev Iberoam ciencias. Puebla, Mexico. 2017;4(2):1-10. Available from: http://www.reibci.org/ publicados/2017/abr/2200103.pdf

21. Rodriguez L, Guevara F, La O M, Romero E, Hernández V, Salas M. Efecto del aluminio en el crecimiento y contenido de clorofila en 
plantulas de maiz. Rev Fitotec Mex. Chiapas. Mexico. 2021;44(1):25-31. https://revfitotecnia. $\mathrm{mx} /$ index.php/RFM/article/view/845/802

22. Francisco S. Evaluación del rendimiento y valor nutricional de dos variedades y dos híbridos de maíz (Zea mays) como forraje en el Valle de Huaral. Universidad Nacional José Faustino Sánchez Carrión. Huacho, Peru. 2019. http://repositorio.unjfsc.edu.pe/handle/ UNJFSC/5084

23. Barrios A. Evaluación del cultivo de maíz (Zea mayz L), a la aplicación de dosis de abonos orgánicos, bajo el sistema de labranza mínima, en la zona de Vinces, durante la época seca. Universidad de Guayaquil, Ecuador. 2014. http://repositorio.ug.edu.ec/handle/ redug/10446

24. Aguilar R, Arévalo CG, Morales A, Galecio M, García PP, Ortiz JQ, et al. Reducción de la fertilización sintética con composta y optimización del riego sobre pudrición del talo (Fusarium spp.) del maíz en sinaloa. Sci Agropecu. 2020;5(4):121-33. http://dx.doi. org/10.17268/sci.agropecu.2014.03.02

25. Del Castillo A. Efecto del compost de residuos sólidos biodegradables, obtenidos del comedor universitario de la UNAS en el Rendimiento Del Cultivo De Maíz (Zea Mays L.) variedad Marginal $28-T$. Universidad Nacional Agraria de la Selva; 2019. http://repositorio. unas.edu.pe/handle/UNAS/1434
26. Romero A, Suarez E, Macias M, Gomez $Y$, Lozno L. Diseño experimental para la obtención de compost apto para uso agrícola a partir de lodo papelero Kraft. Espacios. 2017; 38:1-14. http://hdl.handle.net/11323/2235

27. Tiquia SM, Richard TL, Honeyman MS. Carbon, nutrient, and mass loss during composting. Nutr Cycl Agroecosystems. 2002;62(1):15-24. https://www.researchgate. net/publication/242539803_Carbon_nutrient_ and_mass_loss_during_composting/citation/ download

28. CremonaM, EnriquezA.Algunaspropiedades del suelo que condicionan su comportamiento: el $\mathrm{pH}$ y la conductividad electrica. Presencia. Argentina 2020;73:5-8. https://inta.gob.ar/ documentos/revista-presencia- $n^{\circ}-73$

29. López W, Reynoso R, López J, Villar B, Camas R, García JO. Caracterización físico-química de suelos cultivados con maíz en Villaflores, Chiapas. Rev Mex Ciencias Agrícolas. 2019;10(4):897-910. https://doi.org/10.29312/ remexca.v10i4.1764

30. Cruz WO, Rodríguez LA, Salas MA, Hernández V, Campos RA, Chávez $\mathrm{MH}$, et al. Effect of organic matter and cation exchange capacity on the acidity of soils cultured with corn in two regions of Chiapas, Mexico. Terra Latinoam. 2020;38(3):475-80. https://doi. org/10.28940/terra.v38i3.506 